\title{
Get Well Soon Card: Self Help Program to Overcome Hospital Based Financial Barrier
}

\author{
* Sharad Hari Gajuryal, ${ }^{1}$ Narayan Satyal, ${ }^{2}$ Sangita Bidari, ${ }^{3}$ Shristi Panta, ${ }^{3}$ Basant Pant ${ }^{4}$ \\ ${ }^{1}$ Deputy Medical Director, ${ }^{2}$ Operations Manager, ${ }^{3}$ Project Coordinator, ${ }^{4}$ Chairman and Head of Department; \\ Annapurna Neurological Institute \& Allied Sciences, Maitighar, Kathmandu, Nepal
}

*Corresponding Author: Dr. Sharad Hari Gajuryal; Email : sharad2005@hotmail.com

\begin{abstract}
Background: The health care cost is increasing not only in developed countries but also in developing countries. Although the modality of treatment has made significant progresses over the years, delivery of treatment is sometimes beyond the expectation of patient as well as inaccessible financially. Citizen from developing countries like Nepal have to pay out of their own pocket for even minor health issues. We initiated a new concept to bridge this gap between treatments and necessity of funds through crowd funding scheme.
\end{abstract}

Methods: This was a cross sectional prospective and observational study conducted at Annapurna Neurological Institute and Allied Sciences from July 2017 to April 2020. This study was conducted on "Get Well Soon "card which was a friendly gesture with monetary value provided by visitors to patients. This card was made available in hospital pharmacy without any hidden cost. A convenient sample survey on 100 participant including admitted patients as well as visitors in the hospital was conducted on their perception about Get well soon card before initiating this program.

Results: This innovative self-help scheme has been proven to be quiet successful in a short period of time with many supporters advocating in its favor. Total number of 1012 patients have been benefited from the card which was provided to them by their visitors and relatives during their hospitalization with purchase of card worth NRs. 1144500 ( $\$ 11445)$ during the study period.

Conclusion: Get Well Soon Card can be beneficial to those populations who are financially constrained and lack insurance for the treatment. This Cultural Revolution of crowd funding can be duplicated not only in Nepal, but also in different developing countries where out of pocket system is still prevailing.

Key Word: Crowd funding; Get well soon card; Health care financing; Self-help scheme

\begin{tabular}{|c|c|c|}
\hline \multicolumn{2}{|c|}{ Access this article Online } & Article Info. \\
\hline Quick Response Code & Website: & How to cite this article in Vancouver Style? \\
\hline & www.jkahs.org.np & $\begin{array}{l}\text { Gajuryal S, Satyal N, Bidari S, Panta S, Pant B. GET } \\
\text { WELL SOON CARD: Self Help Program to Over- } \\
\text { come Hospital based Financial. Journal of Karnali } \\
\text { Academy of Health Sciences 2020;3(2): 140-146 }\end{array}$ \\
\hline & $\begin{array}{l}\text { DOI: } \\
\text { https://doi.org/10.3126/ } \\
\text { jkahs.v3i2.31189 }\end{array}$ & $\begin{array}{ll}\text { Received } & : 9 \text { April } 2020 \\
\text { Accepted } & : 10 \text { August. } 2020 \\
\text { Published Online } & : 10 \text { August. } 2020\end{array}$ \\
\hline \& Vier & & $\begin{array}{l}\text { Conflict of Interest : None } \\
\text { Source of Support }: \text { None }\end{array}$ \\
\hline
\end{tabular}




\section{BACKGROUND}

Health care costs are rising day by day and so are the hospitals that provide those services. But with the developing countries, in case they suffer from any diseases, people have to spend their life savings and even go bankrupt to get the desired healthcare service for catastrophic health expenditure. Most of the people in low income countries pay a high proportion of their health costs directly to health care providers out of their own pockets. ${ }^{1-3}$ High levels of out-ofpocket spending for health care have a variety of harmful effects. Some people are deferred from using health services or from continuing treatment because they cannot afford to pay. People who use services may need to cut spending on basic needs such as food, clothing, housing and children's education to meet health costs. Each year, approximately 150 million people experience financial catastrophe, meaning they are obliged to spend on health care more than 40 $\%$ of the income available to them after meeting their basic needs. $^{3}$

Many developing and low-middle income countries are trying to overcome this barrier by introducing a social health protection system, a risk pooling system, that allows a large group of people to share the risk that they may need expensive health care either by tax funded health financing or social health insurance or private insurance method. ${ }^{4}$ Social health insurance which was introduced in 2015 in Nepal seems challenging considering present poverty level, awareness regarding need and working modality of health insurance among the general population and availability and access to quality health care services. Also the health sector budget could not achieve even gainful results due to mismatch in policy and policy implementation despite of political commitment. ${ }^{5}$ Medical crowd funding is rapidly growing in many countries and is seen by many people as a way to cope with government cuts on public health financing and reducing the some limit of financial barrier on healthcare as well as helping the needy. ${ }^{6}$

Nepal is among the poorest countries in the world, and ranked 149 on the Human Development Index of 189 countries during the year 2017.(5)The public health system of Nepal comprises health clinics, health posts, and primary health-care centers at the primary level, district and zonal hospitals at the secondary level, and regional and central hospitals at the tertiary level. Private health-care institutions in the form of hospitals, medical colleges and nursing homes have also emerged in the recent past. Nepal Living Standards Survey indicates that households spend around $5.5 \%$ of their total consumption expenditure on health care with the average per capita out-of-pocket spending on health in Nepal increased sevenfold in nominal terms between 1995- 1996 and 2010- 2011. ${ }^{6}$ hus, people seek less healthcare services in case they were low on finances.

In day to day operations, Hospital administration including finance department have to deal with a lot of patients from poor economic backgrounds requesting for discounts as they were not able to pay the hospital bills. Around $25-30 \%$ of total admitted patients undergone various procedures in our institute usually request the administration for financial support during hospitalization. So we thought that there should be a win-win situation for all the parties involved. We introduced a new program, "Get Well Soon "card, a friendly gesture with a money value that would be given by the visitors to the patients who are currently admitted in the hospital. They aid towards making the economically disadvantaged population get health services without the financial burden.

\section{MATERIALS AND METHODS}

Thiswasaprospectivecrosssectionalandobservational study conducted at Annapurna Neurological Institute and Allied Sciences from July 2017 to April 2020. Our team came up with an idea of financing the patients' health care costs through their well-wishers. Before initiating the program, a convenient sampling survey on 100 participant including admitted patients as well as visitors was conducted on their perception about Get well soon card. Among 100 participant variables like sociodemographic factor, their opinion on card as well as their feedback was assessed. "Get Well Soon "card program had a monetary value of NRs. $250(\sim 2.50)$, NRs. $500(\sim 5.00)$ and NRs. 1000 $(\sim 10.00)$ which was provided by visitors to patients and the collected card amount was to be deducted 
from patient's final bill during discharge. This card was made available in hospital pharmacy without any hidden cost. All the cards sold to visitors were given a unique identification to restrict the duplication of card. The availability of the card and program was disseminated to the public and visitors arriving hospital through various posters and pamphlets that are placed in different area of hospital. Individual data regarding the total number of card provided to the patient were recorded in excel with detail description like amount of card purchased, number of card purchased with detail of patient. Descriptive statistics including frequency and percentage was used to analyze the data and the result was expressed in figure and tabular form.

\section{RESULT}

Before implementing the program, our team took a short survey with the health services seekers in the hospital involving patient, visitors including family members and relatives to understand their opinion for this program. Table 1 shows the basic sociodemographic profile of the participants. Among the total health service seekers $(n=100)$, majority of the participants were visitors $(n=68)$ followed by patient themselves $(n=32)$. The number of male responders $(n=70)$ were higher than female responders $(n=30)$ and majority of participants were from age group 3544 years $(n=39)$.

Table 1: Socio-demographic Profile of Health service seekers $(n=100)$

\begin{tabular}{|l|l|l|}
\hline \multicolumn{1}{|c|}{ Variables } & $\begin{array}{c}\text { Number of } \\
\text { participants }\end{array}$ & $\begin{array}{c}\text { Percentage } \\
\text { (\%) }\end{array}$ \\
\hline Identity & 32 & 32 \\
\hline Patient & 68 & 68 \\
\hline Visitors & & \\
\hline Literacy & 68 & 68 \\
\hline Literate & 32 & 32 \\
\hline Illiterate & & \\
\hline Occupation & 20 & 20 \\
\hline Unskilled & 31 & 31 \\
\hline Semikilled & 36 & 36 \\
\hline Skilled & 13 & 13 \\
\hline Highly Skilled & & \\
\hline Age (in years) & 12 & 12 \\
\hline $15-24$ & 19 & 19 \\
\hline $25-34$ & 39 & 39 \\
\hline $35-44$ & & \\
\hline
\end{tabular}

\begin{tabular}{|l|l|l|}
\hline $45-54$ & 18 & 18 \\
\hline $55-64$ & 12 & 12 \\
\hline 65 and above & 10 & 10 \\
\hline Gender & & \\
\hline Male & 70 & 70 \\
\hline Female & 30 & 30 \\
\hline
\end{tabular}

Regarding the Health service seekers opinion on GET WELL SOON Card, Table 2 shows that Majority of participant agreed that this program will help the patient/ family for their treatment $(\mathrm{n}=88)$, they are willing to buy this card in future $(n=92)$ and they will inform about this program to others $(n=94)$. A very less number of participant disagreed with effective success of this program $(n=3)$ and supporting this program in future $(\mathrm{n}=3)$.

Table 2: Health Service seekers opinion on Get well soon card

\begin{tabular}{|c|c|c|}
\hline Opinion & Response & Frequency \\
\hline $\begin{array}{l}\text { Do you think this } \\
\text { program will help } \\
\text { patient/ family for the } \\
\text { treatment? }\end{array}$ & $\begin{array}{l}\text { Yes } \\
\text { No } \\
\text { Don't Know }\end{array}$ & $\begin{array}{l}88 \\
3 \\
9\end{array}$ \\
\hline $\begin{array}{l}\text { Do you want to buy } \\
\text { GET WELL SOON } \\
\text { CARD in future? }\end{array}$ & $\begin{array}{l}\text { Yes } \\
\text { No } \\
\text { Don't Know }\end{array}$ & $\begin{array}{l}92 \\
3 \\
5\end{array}$ \\
\hline $\begin{array}{l}\text { Do you want to inform } \\
\text { others about this card? }\end{array}$ & $\begin{array}{l}\text { Yes } \\
\text { No } \\
\text { Don't Know }\end{array}$ & $\begin{array}{l}94 \\
0 \\
6\end{array}$ \\
\hline
\end{tabular}

Table 3 expedites the various feedback and suggestions from the respondents before implementing Getwell Soon card program. 82 out of 100 participants provided feedback and suggestions whereas remaining form were left blank. As majority of the participants had positive response to this program, all of the suggestions were genuine and helped the program coordinators to smoothen the program by implementing their suggestion. As this program was initiated for the first time and was challenging in nature, some of the suggestions could not be implemented in the primary phase due to financial constrains as this program was not related to financial benefit. Suggestions including S.N 1, 2,3,5,9 of Table 3 was implemented with initiation of the program.

Table 3: Health Service Seekers feedback/ 


\section{Suggestions on this program}

\begin{tabular}{|c|c|}
\hline SN & Feed back /Suggestions \\
\hline 1 & Get Well Soon Card should not be compulsory to all the patients. ( shall be optional to visitors) \\
\hline 2 & Hospital should not deduct any monetary amount from the card. \\
\hline 3 & Visitors should be well informed about this program. \\
\hline 4 & Advertisement of this card should be done in different media so that general public get well informed. \\
\hline 5 & Card should be given to the patients during discharge as a memory. \\
\hline 6 & The monetary value of card should be in lower denominations as well. \\
\hline 7 & Card should be available in different outlet of Cities including shops and departmental store. \\
\hline 8 & $\begin{array}{l}\text { There must be provision of buying the card from abroad online so that near and dear one can get it } \\
\text { purchased from abroad in the name of patient. }\end{array}$ \\
\hline & There should be space to write personal message to the patient in the card. \\
\hline
\end{tabular}

\section{Our Experience in $\mathbf{3 3}$ months of the program}

With the innovative ideas, support and suggestions of the health service providers and health service seekers, Get well soon card was started soon after the survey at Annapurna Neurological Institute and Allied Sciences with technical support of its sister organization "Family Health Nepal" for the first time in Nepal.The response from the survey was overwhelmingly in favor as it would stop the visitors from bringing flower bouquets and other fruits and drinks but rather contribute directly for the patient's care with a message in it. The card worth monetary value of NRs. 250,NRs.500 \&NRs.1000 $(\sim \$ 2.5, \sim \$ 5 \& \sim \$ 10)$ was made available in Hospital Pharmacy. Information of the card and its benefit was disseminated to the patient and visitors from Media i.e Newspaper, television and social media as well as from posters and pamphlets pasted in visible areas in Hospital. Though there was a slow start, the program quickly picked up its pace as it was a blessing for people from low economic condition getting the treatment with the help of their well-wishers and relatives through collective financing. We monitored the amount of cards that were being sold through the pharmacy and the people getting those cards from their relatives. The hospital is also generating less wastes as visitors are bringing in less fruits, drinks and plastic. There was a total support for this program and everyone perceived it as a great concept of crowd-funding. Total number of 1012 patients have already been benefited from the card with purchase of card worth NRs. $1144500(\sim \$ 11445)$ till date. All the patients and their families who got benefited from the card mostly had a financial hardship for the payment of their treatment which was partly helpful from get well soon card. Number of patients requiring financial support from hospital were happily supported by this crowd funding scheme from their own relatives resulting in at least a sufficient aid for financial settlement during hospitalization. As around 25$30 \%$ of admitted patient usually request our financial section for discount on their treatment, this scheme also somehow reduced the patients with financial hardship for requesting hospital administration to waive some part of their treatment charge.

Figure 1 shows the total number of card purchased by visitors for financing the patient according to the denomination of card type. The majority of visitors/ relatives purchased card worth NRs.500 or $\sim \$ 5.0$ ( $\mathrm{n}=826,28 \%$ ) to their patients followed by card worth NRs. 250 or $\$ 2.5(\mathrm{n}=524,53 \%)$ and card worth Rs.1000 or $\$ 10.0(\mathrm{n}=230,19 \%)$. The purchasing amount of get well soon card increased by time, the information of which has been provided in figure 2 with total of NRs. 1144500 ( $\$ 11445)$ within the 32 months of initiation of this program. Figure 3 shows the kind of cards available with us for the program. 
Figure 1: Number of Get well soon card purchased by visitors according to card type

\section{Get Well Soon Card Purchased $(n=2182)$}

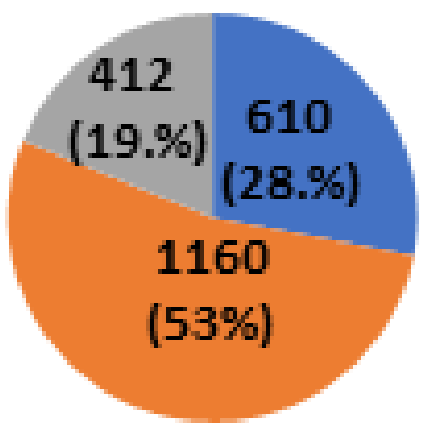

NRs. 250

NRs. 500

NRs. 1000

Figure 2: Amount of Get Well Soon card purchased by visitors in given periods

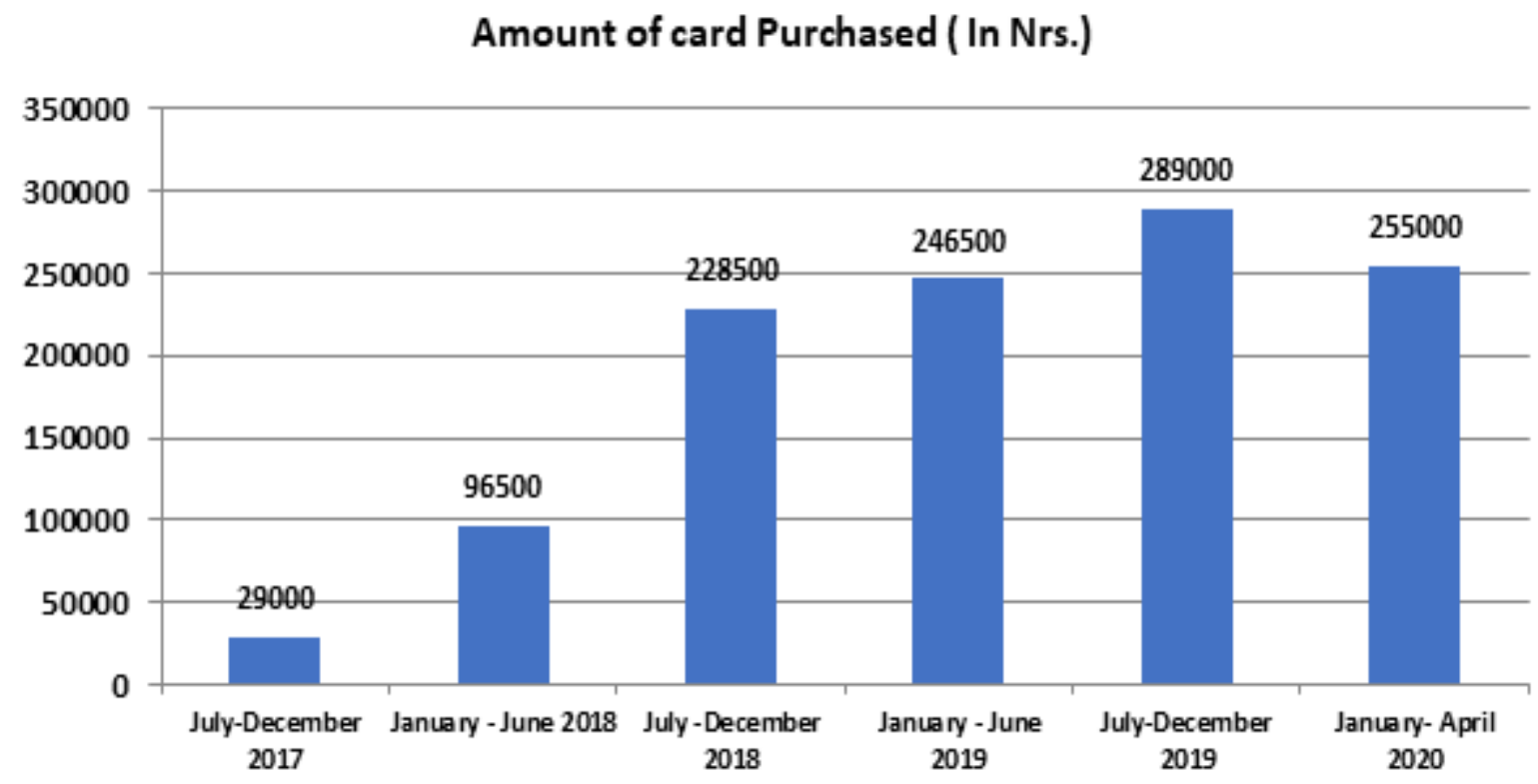

Figure 3: Different types of Get Well Soon Card (Nrs. 1000, Nrs. 500 \& Nrs. 250 Respectively) Challenges and future aspect:
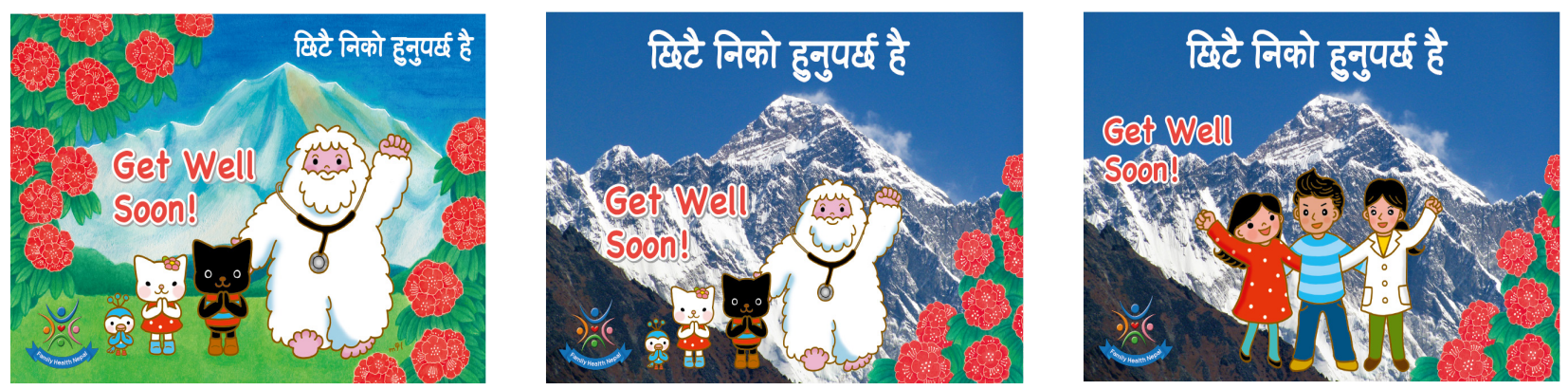
Health care costs are rising day by day and so are the hospitals that provide those services. But in the developing countries, in case they suffer from any diseases, people have to spend their life savings and even go bankrupt to get the desired healthcare service.

Regarding the challenges, this program was largely unknown to the general population in its initial stages as we did not conduct extensive marketing, and people would still come with fruits and juices while coming to visit their relatives. In context of Nepali Culture, the concept of giving fruits, flowers and other edibles when someone gets admitted in hospital instead of money is highly prevalent and it can be uncomfortable and even awkward for patients to talk about get well soon card to their visitors as it would be like asking for money directly. Though this program is a innovative concept and a self-help scheme for patient as well as aid in quality care for the patient, this program may not be properly endorsed by different hospitals because of lack of profitability .Even for the logistic, printing and human resource cost, it is a challenging part for organizations for delivering this program within the Institute. Similarly, this program couldn't be extended to the outlets far from the hospital as it requires extensive networks and couldn't be applied in other hospitals as a medium of payment because of lack of initiations of similar program in other hospitals.

The program executors further came up with the solution for future expansion and recommendation for this program to make it more success that is affordable to every individual. With proper collaboration, Get well Soon card can be expanded to other hospitals as well so that same card could be used as a medium of payment in different hospital by making them available in different outlets of the city. There shall be a provision of online purchase of the card via digital payment gateways for the ease of purchasers and receiver of get well soon card. As we found that some individual providing a help of few thousands to same patients, higher denomination of card shall be available in the outlets in near future. There must be a government endorsement of this program so that this could be available in public hospital as well with a mass dissemination of information through media about the benefit of this card to patients, their relatives as well as for the health care organizations.

\section{DISCUSSION}

Medical crowd funding is an increasingly common response to overwhelming healthcare costs and is often a resource for both instrumental and emotional social support. ${ }^{7}$ In the country like Nepal where there are lots of challenges in implementation government insurance system with very minimal ceiling amount as well as large proportion of withdrawal rate by insured member ${ }^{5}$, this self-help program can be beneficial to the patients who can be helped financially by their own relatives.

We have successfully implemented an affordable, innovative system of self-help program in our Institute which was positively endorsed and accepted by the health service seekers with a great sense of satisfaction and feedback. This may be due to optional help to the patients instead of providing them edibles and gifts from the visitors. We are now refining and expanding Get Well Card system in more systematic level so that it can be accepted by all the health care organizations in the country.

The future expansion aspect of Get well soon card still remains questionable as it should be accepted by all the health care organizations in Nepal to provide this quality services to all the patient in our setting. In particular, a set of marketing, sponsors, its importance and its positive impact on patient and health service seekers should be disseminated to the respective health care organizations in the country which shall make it acceptable in the health care institutes countrywide. Though some of the health care centers have already duplicated similar type of programs in Nepal, if this program can be properly accepted by all public and private health care sectors, it can be a great example of self-help program specially in poor and developing countries which lacks public health insurance system. It is unquestionable, after all, this self-help scheme are the part of health care financing in $21^{\text {st }}$ century health care system, yet many open questions remain as to what the acceptance by all the health care organizations actually looks like. 


\section{CONCLUSION}

Get Well Soon Card is a one of the innovative way crowd funding as well as self-help scheme and is beneficial to that population who are not insured and who lacks the necessary financial recourses for the treatment during hospitalization. This Cultural Revolution has been started from a single Institute and shall be duplicated not only in various public and private health care organizations of Nepal as well as in different developing countries where out of pocket system is still prevailing.

Acknowledgements: The Author would like to acknowledge Annapurna Neuro Pharmacy staffs especially to Jayaram Adhikari, Ashok Bhusal and Dipesh Dahal for their support and contribution in making this program successful.

\section{REFERENCES}

1. Escobar ML, Griffin CC, Shaw RP . The Impact of Health Insurance in Low-and Middle-Income Countries .BROOKINGS INSTITUTION PRESS [Internet]. [cited 2019 Apr 10]. Full Text

2. Han W. Health care system reforms in developing countries. J Public health Res 2012;1(3):199207. PubMed Google Scholar CrossRef
3. Azzani M, Roslani AC, Su TT. Determinants of Household Catastrophic Health Expenditure: A Systematic Review. Malays J Med Sci. Jan-Feb 2019; 26(1): 15-43 PubMed CrossRef Google $\underline{\text { Scholar }}$

4. Mathauer, I., Saksena, P. \& Kutzin, J. Pooling arrangements in health financing systems: a proposed classification.2019 International Journal of Equity Health 18, 198 Full Text CrossRef

5. MoHP. Review of Social Health Insurance Scheme in Selected Districts of Nepal - Nepal Health Research Council 2018. Full Text

6. Bassani, G., Marinelli, N. \& Vismara, S. Crowd funding in healthcare. Journal of Technology Transfer 2018; 44, 1290-1310 Full text

7. Gonzales AL, Kwon EY, Lynch T, Fritz N. Better everyone should know our business than we lose our house: Costs and benefits of medical crowdfunding for support, privacy, and identity. New Media Soc 2018; 20(2):641-58. CrossRef Google Scholar Full Text 